\title{
PET e SPECT cerebrais na avaliação dos estados demenciais
}

\author{
Carlos Alberto Buchpiguel
}

Departamento de Radiologia da Faculdade de Medicina da USP

\section{Introdução}

Os estados de deterioração cognitiva se caracterizam por impor sérias limitações sociais e econômicas à sociedade como um todo. À medida que a expectativa de vida aumenta com os avanços da medicina, mais freqüente torna-se o diagnóstico de estados demenciais. Nos Estados Unidos, por exemplo, têm-se confirmado mais de quatro milhões de casos da doença de Alzheimer diagnosticados, o que, por sua vez, produz custos anuais de, aproximadamente, 70 bilhões de dólares. ${ }^{1,2}$ Portanto, torna-se imperativo que testes diagnósticos mais precisos sejam aplicados, quer no sentido de reduzir custos por um diagnóstico incorreto, quer por permitir que um número mais significativo de doentes tenha acesso ao tratamento com novas drogas, em fase mais precoce.

Estudos funcionais de neuroimagem se caracterizam por apresentar alterações funcionais. Estes se correlacionam muito intimamente com o espectro fisiopatológico que domina essas desordens bem antes que sintomas específicos possam estar presentes. Estudos com tomografia por emissão de pósitrons têm sido investigados desde a década de $80,{ }^{3,4}$ porém, apenas recentemente os dados têm sido reavaliados de forma crítica e sistemática. ${ }^{5,6} \mathrm{O}$ padrão funcional que mostra maior associação com o diagnóstico de demência do tipo Alzheimer é o déficit de metabolismo ou de fluxo sangüíneo regional em topografia do córtex entorrinal (temporal) com extensão para região parietal bilateral (córtex de associação) (Figura).

Embora esse padrão tenha se mostrado bastante sensível, seja utilizando tomografia por emissão de pósitron (PET) ou tomografia por emissão de fóton único (SPECT), percebe-se que a especificidade pode variar entre a faixa dos $60 \%$ a $70 \%$, principalmente quando casos de diagnóstico clínico mais complexo são incluídos na análise. Muitos dos trabalhos iniciais avaliaram a acurácia dos estudos funcionais em relação ao diagnóstico clínico baseado nos critérios do National Institute of Neurological and Communicative Disorders and Stroke/ Alzheimer's disease and Related Disorders Association (NINCDS-ADRDA). Os trabalhos mais recentes promovem a avaliação crítica do papel dos estudos funcionais de neuroimagem, avaliando principalmente pacientes com diagnóstico clínico inconclusivo e que possuem confirmação histológica para verificação da sensibilidade, especificidade, valores de predição e acurácia do método. Embora alguns estudos tenham mostrado de forma preliminar associação direta entre densidade de bandas neurofibrilares e déficit metabólico têmporo-parietal posterior bilateral, artigo publicado por Mielke em 1996 demonstrou não haver qualquer relação quantitativa entre presença de placas senis e bandas neurofibrilares com padrões funcionais ditos típicos na PET. $^{7}$ Um estudo recente, analisando 22 pacientes com demência que possibilitaram posterior análise histológica do cérebro mostrou que, enquanto o critério clínico de doença de Alzheimer provável apresentou uma especificidade de $100 \%$, o mesmo mostrou baixa sensibilidade $(63 \%)$. Por outro lado, a presença de hipometabolismo na região parieto-temporal bilateral pela PET mostrou sensibilidade de $93 \%$, especificidade de $63 \%$ e acurácia de $82 \% .^{8}$ É importante referir que esse trabalho apenas selecionou casos onde o diagnóstico clínico era complexo ou inconclusivo e, portanto, pode caracterizar uma tendência de subestimar a especificidade do estudo funcional. Considerando os resultados apresentados por esse grupo, semelhantes a metanálises agrupando estudos que correlacionaram os resultados da PET com histologia, percebe-se que, nos casos mais difíceis de se diagnosticar precisamente o quadro demencial, o uso de métodos de imagem funcional permitiria diagnosticar cerca de $20 \%$ mais casos do que se diagnosticaria utilizando exclusivamente o exame clínico. Igualmente, além do valor diagnóstico, tem sido discutido o valor prognóstico desses métodos. Por exemplo, quando se verifica um maior grau de déficit metabólico ou de fluxo regional, estima-se um declínio cognitivo mais rápido e severo. ${ }^{9}$

Com SPECT existem igualmente vários estudos mostrando correlação com análise histológica. Jagust mostrou, estudando 11 pacientes com demência de Alzheimer, que a redução de fluxo em região têmporo-parietal bilateral foi eficaz na diferenciação entre demência do tipo Alzheimer e outros tipos de demência. ${ }^{10}$

Read et al correlacionaram os resultados de SPECT em 27 pacientes portadores de demência com os resultados da autópsia e mostraram sensibilidade de $96 \%$, contra $74 \%$ para o exame clínico. ${ }^{11}$ Contudo, existem relatos no nosso meio de sensibilidades mais baixas do SPECT na detecção de padrões funcionais típicos. Buchpiguel et al, analisando 52 pacientes com diagnóstico de doença de Alzheimer e 11 voluntários normais, mostraram sensibilidade de aproximadamente $70 \%$ e especificidade de $100 \% .{ }^{12}$ A menor sensibilidade poderia estar associada a amostragem populacional mais heterogênea ou complexa, ou mesmo a fatores técnicos como uso de equipamento de limitada resolução espacial, uso de interpretação qua- 
litativa em vez de quantitativa etc. Em fase posterior, o mesmo grupo mostrou, analisando 104 pacientes com critério provável para demência de Alzheimer, que o déficit temporal e parietal posterior estava mais fortemente associado a grupos de pacientes na fase pré-senil, com maior grau de severidade do declínio cognitivo, e em pacientes do sexo masculino. Duração dos sintomas, tipo de câmara de cintilação ou mesmo radiofármaco empregado não influenciaram os resultados em qualquer sentido. ${ }^{13}$

Porém, qual a relevância da indicação de métodos funcionais mais sensíveis de neuroimagem em pacientes com esse tipo de desordem degenerativa cerebral? Mais de duas décadas atrás, não existiam perspectivas de tratamento medicamentoso para esse tipo de desordem orgânica. Contudo, nos últimos anos, tem-se verificado um significativo progresso no arsenal terapêutico, com os inibidores da colinesterase. Percebe-se hoje que pacientes diagnosticados em fase precoce podem ter seu declínio cognitivo e deterioração intelectual retardados com uso dessas drogas. ${ }^{14}$ Portanto, não apenas do ponto de vista prognóstico, é possível utilizar esses métodos para selecionar mais adequadamente os pacientes que podem eventualmente se beneficiar de um tratamento precoce com drogas. Há, entretanto, carência de estudos prospectivos na literatura demonstrando, de forma efetiva, o impacto da aplicação desses métodos funcionais na qualidade de vida e no aumento da sobrevida nesse tipo de condição neuropatológica.

\section{Referências}

1. Evans DA. Estimated prevalence of Alzheimer's disease in the US. Milbank Q 1990;68:267-89.

2. Ernst RL, Hay JW. The US economic and social costs of Alzheimer's disease revisited.Am J Pub Health 1994;84:1261-4.

3. Farkas T, Ferris SH, Wolf AP, De Leon MJ, Christman DR, Reisberg B, et al. 18F-2-dexy-2-fluoro-D-glucose as a tracer in the positron emission tomographic study of senile dementia. Am J Psychiatr 1982;139:352-3.

4. Benson DF, Kuhl DE, Phelps ME, Cummings JL, Tsai SY. Positron emission computed tomography in the diagnosis of dementia. Trans Am Neurol Assoc 1981;106:68-71.

5. Silverman DHA, Small GW, Phelps ME. Clinical value of neuroimaging in the diagnosis of dementia: sensitivity and specificity of regional cerebral metabolic and other parameters for early identification of Alzheimer's disease. Clin Positron Imaging 1999;2:119-30.

6. Mazziotta JC, Frakowiak RSJ, Phelps ME. The use of positron emission tomography in the clinical assessment of dementia. Semin Nucl Med 1992;22:233-46.

7. Mielke R, Schroder, Fink GR, Kessler J, Herholz K, Heiss WD. Regional cerebral glucose metabolism and postmortem pathology in Alzheimer's disease. Acta Neuropathol 1996;91:174-9.
8. Hoffman JM, Welsh-Bohmer KA, Hanson M, Crain B, Hulette C, Earl $\mathrm{N}$, Coleman ER. FDG PET imaging in patients with pathologically verified dementia. J Nucl Med 2000;41:1921-8.

9. Small GW, Ercoli LM, Silverman DHS, et al. Cerebral metabolic and cognitive decline in persons at genetic risk for Alzheimer's disease. Proc Nat Acad Sci USA 2000;97:6037-42.

10. Jagust WJ, Reed BR, Ellis WG, Eberling JL, Budinger TF. Single photon emission computed tomographic perfusion imaging in autopsydiagnosed dementia. J Neuroimag 1993;3:93-9.

11. Read SL, Miller BL, Mena I, Kim R, Habashi H, Darby A. SPECT in dmentia: clinical and pathological correlation. J Am Geriatr Soc 1995;43:1243-7.

12. Buchpiguel CA, Mathias SC, Itaya LY, Barros NG, Portela LA, Freitas $\mathrm{JM}$, et al. Brain Spect in dementia. A clinical-scintigraphic correlation. Arq Neuropsiquiatr 1996;54:375-83.

13. Nitrini R, Buchpiguel CA, Caramelli P, Bahia VS, Mathias SC, Nascimento CM, et al. Spect in Alzheimer's disease: features associated with bilateral parietotemporal hypoperfusion. Acta Neurol 2000;101:1-5.

14. Rogers SL, Farlow MR, Doody RS, Mohs R, Friedhoff LT. A 24-week, double-blind, placebo-controlled trial of donepezil in patients with Alzheimer's disease. Donepezil Study Group. Neurol 1998;50:136-45.

Correspondência: Carlos Alberto Buchpiguel

Rua Alberto Faria, 1570 - 05459-001 São Paulo, SP 